JOURNAL OF THE

CHUNGCHEONG MATHEMATICAL SOCIETY

Volume 26, No. 4, November 2013

http://dx.doi.org/10.14403/jcms.2013.26.4.707

\title{
STABILITY OF FUNCTIONAL EQUATION AND INEQUALITY IN FUZZY NORMED SPACES
}

\author{
HARK-MAhn KIM* AND YANG-Hi LEE**
}

\begin{abstract}
In this paper, we investigate a fuzzy version of stability theory for the following functional equation
\end{abstract}

$$
f(x+y)+f(x-y)-2 f(x)-f(y)-f(-y)=0
$$

in the sense of M. Mirmostafaee and M. S. Moslehian.

\section{Introduction and preliminaries}

A classical question in the theory of functional equations is "when is it true that a mapping, which approximately satisfies a functional equation, must be somehow close to an exact solution of the equation?". Such a problem, which has been called a stability problem of the functional equation, was formulated by S. M. Ulam [16] in 1940. In the next year, D. H. Hyers [5] gave a partial solution of Ulam's problem for the case of approximate additive mappings. Subsequently, his result was generalized by T. Aoki [1] for additive mappings, and by Th. M. Rassias [14] for linear mappings, to considering the stability problem with unbounded Cauchy differences. During the last decades, the stability problems of functional equations have been extensively investigated by a number of mathematicians, see $[4,6,10,15]$. In 1984, A. K. Katsaras [8] defined a fuzzy norm on a linear space to construct a fuzzy structure on the space. Since then, some mathematicians have introduced several types of fuzzy norm in different points of view. In particular, T. Bag and S. K. Samanta [2], following that of S. C. Cheng and J. N. Mordeson [3], gave

Received June 25, 2013; Accepted September 27, 2013.

2010 Mathematics Subject Classification: Primary 39B82; Secondary 39B52.

Key words and phrases: fuzzy normed space, fuzzy $q$-almost quadratic-additive mapping, Drygas functional equation.

Correspondence should be addressed to Hark-Mahn Kim, hmkim@cnu.ac.kr.

The author was supported by Basic Research Program through the National Research Foundation of Korea (NRF) funded by the Ministry of Education (No. 2012R1A1A2008139). 
an idea of a fuzzy norm in such a manner that the corresponding fuzzy metric is of Kramosil and Michalek type [9]. In 2008, M. Mirmostafaee and M. S. Moslehian [12] obtained a fuzzy version of stability for the Cauchy functional equation:

$$
f(x+y)-f(x)-f(y)=0 .
$$

In the same year, they [11] proved a fuzzy version of stability theorem for the quadratic functional equation:

$$
f(x+y)+f(x-y)-2 f(x)-2 f(y)=0 .
$$

A solution of (1.1) is called an additive mapping and a solution of (1.2) is called a quadratic mapping.

Now, we consider the functional equation:

$$
f(x+y)+f(x-y)-2 f(x)-f(y)-f(-y)=0,
$$

which is called a Drygas functional equation. A solution of (1.3) is called a solution of the Drygas functional equation, or called a quadraticadditive mapping in this paper, since the equation (1.3) has exactly a Cauchy additive mapping and a quadratic mapping as a general solution. A stability result for the Drygas functional equation (1.3) was derived by S.-M. Jung and P.K. Sahoo [7] and generalized by D.L. Yang [17]. In 2008, C.-G. Park [13] obtained a stability of the functional equation (1.3) by taking and composing an additive mapping $A$ and a quadratic mapping $Q$ to prove the existence of a quadratic-additive mapping $F$ which is close to the given mapping $f$.

In this paper, we investigate to get a general stability result of the Cauchy additive and quadratic type functional equation (1.3) in the fuzzy normed linear spaces by the manner of M. Mirmostafaee and M.S. Moslehian [11]. To do this, we introduce a Cauchy sequence $\left\{J_{n} f(x)\right\}$ starting from a given mapping $f$, which converges to the desired mapping $F$ in the fuzzy settings which is a solution of (1.3), so that we can take the desired solution $F$ near by the approximate solution $f$ in the fuzzy settings only at once according to our suggested method in the paper.

\section{Fuzzy stability of the functional equation (1.3)}

We present to use the definition of a fuzzy normed space given in [2] to exhibit a reasonable fuzzy version of stability for the Cauchy additive and quadratic type functional equation in the fuzzy normed linear space. 
Let $X$ be a real linear space. A function $N: X \times \mathbb{R} \rightarrow[0,1]$ (so-called fuzzy subset) is said to be a fuzzy norm on $X$ if for all $x, y \in X$ and all $s, t \in \mathbb{R}$,

(N1) $N(x, c)=0$ for $c \leq 0$;

(N2) $x=0$ if and only if $N(x, c)=1$ for all $c>0$;

(N3) $N(c x, t)=N(x, t /|c|)$ if $c \neq 0$

(N4) $N(x+y, s+t) \geq \min \{N(x, s), N(y, t)\}$;

(N5) $N(x, \cdot)$ is a non-decreasing function on $\mathbb{R}$ and $\lim _{t \rightarrow \infty} N(x, t)=1$.

The pair $(X, N)$ is called a fuzzy normed linear space [2]. Let $(X, N)$ be a fuzzy normed linear space. Let $\left\{x_{n}\right\}$ be a sequence in $X$. Then $\left\{x_{n}\right\}$ is said to be convergent if there exists $x \in X$ such that $\lim _{n \rightarrow \infty} N\left(x_{n}-\right.$ $x, t)=1$ for all $t>0$. In this case, $x$ is called the limit of the sequence $\left\{x_{n}\right\}$ and we denote it by $N-\lim _{n \rightarrow \infty} x_{n}=x$. A sequence $\left\{x_{n}\right\}$ in $X$ is called Cauchy if for each $\varepsilon>0$ and each $t>0$ there exists $n_{0}$ such that for all $n \geq n_{0}$ and all $p>0$ we have $N\left(x_{n+p}-x_{n}, t\right)>1-\varepsilon$. It is known that every convergent sequence in a fuzzy normed space is Cauchy. If each Cauchy sequence is convergent, then the fuzzy norm is said to be complete and the fuzzy normed space is called a fuzzy Banach space.

Let $(X, N)$ be a fuzzy normed space and $\left(Y, N^{\prime}\right)$ a fuzzy Banach space. For a given mapping $f: X \rightarrow Y$, we use the abbreviation

$$
D f(x, y):=f(x+y)+f(x-y)-2 f(x)-f(y)-f(-y)
$$

for all $x, y \in X$. For given $q>0$, the mapping $f$ is called a fuzzy $q$-almost quadratic-additive mapping, if

$$
N^{\prime}(D f(x, y), t+s) \geq \min \left\{N\left(x, s^{q}\right), N\left(y, t^{q}\right)\right\}
$$

for all $x, y \in X$ and all $s, t \in(0, \infty)$. Now, we are ready to get general stability results in the fuzzy normed linear settings.

Theorem 2.1. Let $q$ be a positive real number with $q \neq \frac{1}{2}$, 1 . Suppose that $f$ is a fuzzy q-almost quadratic-additive mapping from a fuzzy normed space $(X, N)$ into a fuzzy Banach space $\left(Y, N^{\prime}\right)$. Then there is a unique quadratic-additive mapping $F: X \rightarrow Y$ such that

$$
N^{\prime}(F(x)-f(x), t) \geq\left\{\begin{array}{c}
\sup _{t^{\prime}<t}\left\{N\left(x, 2^{-q}\left(2-2^{p}\right)^{q} t^{\prime q}\right)\right\} \\
\text { if } q>1 ; \\
\sup _{t^{\prime}<t}\left\{N\left(x, 4^{-q}\left(4-2^{p}\right)^{q}\left(2^{p}-2\right)^{q} t^{\prime q}\right)\right\} \\
\text { if } \frac{1}{2}<q<1 ; \\
\sup _{t^{\prime}<t}\left\{N\left(x, 2^{-q}\left(2^{p}-4\right)^{q} t^{\prime q}\right)\right\} \\
\text { if } 0<q<\frac{1}{2}
\end{array}\right.
$$

for each $x \in X$ and $t>0$, where $p:=1 / q$. 
Proof. It follows from (2.1) and (N4) that

$$
N^{\prime}(f(0), t)=N^{\prime}(D f(0,0), 2 t) \geq N\left(0, t^{q}\right)=1
$$

for all $t \in(0, \infty)$. Thus, we have $f(0)=0$ by (N2).

Now, we will prove the theorem in three cases, $q>1, \frac{1}{2}<q<1$, and $0<q<\frac{1}{2}$.

Case 1. Let $q>1$ and let $J_{n} f: X \rightarrow Y$ be a mapping defined by

$$
J_{n} f(x)=\frac{1}{2}\left[4^{-n}\left(f\left(2^{n} x\right)+f\left(-2^{n} x\right)\right)+2^{-n}\left(f\left(2^{n} x\right)-f\left(-2^{n} x\right)\right)\right]
$$

for all $x \in X$. Notice that $J_{0} f(x)=f(x)$ and

$$
\begin{aligned}
& J_{j} f(x)-J_{j+1} f(x) \\
& =\frac{2^{j+1}-1}{2 \cdot 4^{j+1}} D f\left(-2^{j} x,-2^{j} x\right)-\frac{2^{j+1}+1}{2 \cdot 4^{j+1}} D f\left(2^{j} x, 2^{j} x\right)
\end{aligned}
$$

for all $x \in X$ and $j \geq 0$. Together with (N3), (N4) and (2.1), the last equation implies that for any integers $n, m$ with $n>m \geq 0$ one has

$$
\begin{aligned}
& N^{\prime}\left(J_{m} f(x)-J_{n+m} f(x), \sum_{j=m}^{n+m-1}\left(\frac{2^{p}}{2}\right)^{j} t^{p}\right) \\
& \geq N^{\prime}\left(\sum_{j=m}^{n+m-1}\left(J_{j} f(x)-J_{j+1} f(x)\right), \sum_{j=m}^{n+m-1}\left(\frac{2^{p}}{2}\right)^{j} t^{p}\right) \\
& \geq \min \bigcup_{j=m}^{n+m-1}\left\{N^{\prime}\left(J_{j} f(x)-J_{j+1} f(x),\left(\frac{2^{p}}{2}\right)^{j}\right) t^{p}\right\} \\
& \geq \min \bigcup_{j=m}^{n+m-1}\left\{\operatorname { m i n } \left\{N^{\prime}\left(\frac{-\left(2^{j+1}+1\right) D f\left(2^{j} x, 2^{j} x\right)}{2 \cdot 4^{j+1}}, \frac{\left(2^{j+1}+1\right) 2^{j p} t^{p}}{4^{j+1}}\right),\right.\right. \\
& \geq \min \bigcup_{j=m}^{n+m-1}\left\{N\left(2^{j} x, 2^{j} t\right)\right\}=N(x, t)
\end{aligned}
$$

for all $x \in X$ and $t>0$. Let $\varepsilon>0$ be given. Since $\lim _{t \rightarrow \infty} N(x, t)=1$, there is a positive real number $t_{0}>0$ such that

$$
N\left(x, t_{0}\right) \geq 1-\varepsilon \text {. }
$$

We observe that for some $\tilde{t}>t_{0}$ the series $\sum_{j=0}^{\infty}\left(\frac{2^{p}}{2}\right)^{j} \tilde{t}^{p}$ converges for $p=\frac{1}{q}<1$. Thus, it guarantees that, for an arbitrary given $c>0$, there 
exists a positive integer $n_{0}>0$ such that

$$
\sum_{j=m}^{n+m-1}\left(\frac{2^{p}}{2}\right)^{j} \tilde{t}^{p}<c
$$

for each $m \geq n_{0}$ and all $n>0$. Therefore, by (N5) and (*), we have

$$
\begin{aligned}
& N^{\prime}\left(J_{m} f(x)-J_{n+m} f(x), c\right) \\
& \geq N^{\prime}\left(J_{m} f(x)-J_{n+m} f(x), \sum_{j=m}^{n+m-1}\left(\frac{2^{p}}{2}\right)^{j} \tilde{t}^{p}\right) \\
& \geq N(x, \tilde{t}) \geq N\left(x, t_{0}\right) \geq 1-\varepsilon .
\end{aligned}
$$

for all $x \in X$. Hence $\left\{J_{n} f(x)\right\}$ is a Cauchy sequence in the fuzzy Banach space $\left(Y, N^{\prime}\right)$, and so we can define a mapping $F: X \rightarrow Y$ by

$$
F(x):=N^{\prime}-\lim _{n \rightarrow \infty} J_{n} f(x), \quad x \in X .
$$

Moreover, if we put $m=0$ in the fuzzy inequality $(*)$, we have

$$
N^{\prime}\left(f(x)-J_{n} f(x), t\right) \geq N\left(x, \frac{t^{q}}{\left(\sum_{j=0}^{n-1}\left(\frac{2^{p}}{2}\right)^{j}\right)^{q}}\right)
$$

for all $x \in X$.

Next, we will show that $F$ is the desired quadratic-additive mapping. Using (N4), we have

$$
\begin{gathered}
(2.5) N^{\prime}(D F(x, y), t) \\
\geq \min \left\{N^{\prime}\left(\left(F-J_{n} f\right)(x+y), \frac{t}{10}\right), \quad N^{\prime}\left(\left(F-J_{n} f\right)(x-y), \frac{t}{10}\right),\right. \\
N^{\prime}\left(2\left(J_{n} f-F\right)(x), \frac{t}{10}\right), \quad N^{\prime}\left(\left(J_{n} f-F\right)(y), \frac{t}{10}\right), \\
\left.N^{\prime}\left(\left(J_{n} f-F\right)(-y), \frac{t}{10}\right), \quad N^{\prime}\left(D J_{n} f(x, y), \frac{t}{2}\right)\right\}
\end{gathered}
$$

for all $x, y \in X$ and all $n \in \mathbb{N}$. We observe that the first five terms on the right hand side of (2.5) tend to 1 as $n \rightarrow \infty$ by the definition of $F$ and (N2), and that the last term satisfies the following 


$$
\begin{aligned}
& N^{\prime}\left(D J_{n} f(x, y), \frac{t}{2}\right) \\
& \geq \min \left\{N^{\prime}\left(\frac{D f\left(2^{n} x, 2^{n} y\right)}{2 \cdot 4^{n}}, \frac{t}{8}\right), N^{\prime}\left(\frac{D f\left(-2^{n} x,-2^{n} y\right)}{2 \cdot 4^{n}}, \frac{t}{8}\right),\right. \\
& \left.\quad N^{\prime}\left(\frac{D f\left(2^{n} x, 2^{n} y\right)}{2 \cdot 2^{n}}, \frac{t}{8}\right), N^{\prime}\left(\frac{D f\left(-2^{n} x,-2^{n} y\right)}{2 \cdot 2^{n}}, \frac{t}{8}\right)\right\}
\end{aligned}
$$

for all $x, y \in X$. Thus, using (N3) and (2.1), we obtain

$$
\begin{aligned}
& N^{\prime}\left(\frac{\left.D f\left( \pm 2^{n} x, \pm 2^{n} y\right)\right)}{2 \cdot 4^{n}}, \frac{t}{8}\right) \\
& =N^{\prime}\left(D f\left( \pm 2^{n} x, \pm 2^{n} y\right), \frac{4^{n} t}{4}\right) \\
& \geq \min \left\{N\left(2^{n} x,\left(\frac{4^{n} t}{8}\right)^{q}\right), N\left(2^{n} y,\left(\frac{4^{n} t}{8}\right)^{q}\right)\right\} \\
& \geq \min \left\{N\left(x, \frac{2^{(2 q-1) n}}{2^{3 q}} t^{q}\right), N\left(y, \frac{2^{(2 q-1) n}}{2^{3 q}} t^{q}\right)\right\},
\end{aligned}
$$

and also we have similarly

$$
\begin{aligned}
& N^{\prime}\left(\frac{\left.D f\left( \pm 2^{n} x, \pm 2^{n} y\right)\right)}{2 \cdot 2^{n}}, \frac{t}{8}\right) \\
& \geq \min \left\{N\left(x, \frac{2^{(q-1) n}}{2^{3 q}} t^{q}\right), N\left(y, \frac{2^{(q-1) n}}{2^{3 q}} t^{q}\right)\right\}
\end{aligned}
$$

for all $x, y \in X$ and all $n \in \mathbb{N}$. According to $q>1$ together with (N5), we can deduce that the last term of (2.5) also tends to 1 as $n \rightarrow \infty$. It follows from $(2.5)$ that $N^{\prime}(D F(x, y), t)=1$ for each $x, y \in X$ and $t>0$. This equality means from the fuzzy normed property (N2) that $D F(x, y)=0$ for all $x, y \in X$, and so $F$ is the desired quadratic-additive mapping.

Next we approximate the difference between $f$ and $F$ in the fuzzy sense. For an arbitrary fixed $x \in X$ and $t>0$, choose $0<\varepsilon<1$ and $0<t^{\prime}<t$. Since $F$ is the limit of $\left\{J_{n} f(x)\right\}$, there is a positive integer $n \in \mathbb{N}$ such that

$$
N^{\prime}\left(F(x)-J_{n} f(x), t-t^{\prime}\right) \geq 1-\varepsilon .
$$


By (2.4), we have

$$
\begin{aligned}
& N^{\prime}(F(x)-f(x), t) \\
& \geq \min \left\{N^{\prime}\left(F(x)-J_{n} f(x), t-t^{\prime}\right), N^{\prime}\left(J_{n} f(x)-f(x), t^{\prime}\right)\right\} \\
& \geq \min \left\{1-\varepsilon, \quad N\left(x, \frac{t^{\prime q}}{\left(\sum_{j=0}^{n-1}\left(\frac{2^{p}}{2}\right)^{j}\right)^{q}}\right)\right\} \\
& \geq \min \left\{1-\varepsilon, \quad N\left(x, 2^{-q}\left(2-2^{p}\right)^{q} t^{\prime q}\right)\right\} .
\end{aligned}
$$

Because $0<\varepsilon<1$ is arbitrary, we get the approximate inequality (2.2) in this case.

Finally, to prove the uniqueness of $F$, let $F^{\prime}: X \rightarrow Y$ be another quadratic-additive mapping satisfying the approximation (2.2). Then by (2.3), we get

$$
\left\{\begin{array}{l}
F(x)-J_{n} F(x)=\sum_{j=0}^{n-1}\left(J_{j} F(x)-J_{j+1} F(x)\right)=0 ; \\
F^{\prime}(x)-J_{n} F^{\prime}(x)=\sum_{j=0}^{n-1}\left(J_{j} F^{\prime}(x)-J_{j+1} F^{\prime}(x)\right)=0
\end{array}\right.
$$

for all $x \in X$ and all $n \in \mathbb{N}$. Together with (N4) and (2.2), this implies that

$$
\begin{aligned}
& N^{\prime}\left(F(x)-F^{\prime}(x), t\right) \\
& =N^{\prime}\left(J_{n} F(x)-J_{n} F^{\prime}(x), t\right) \\
& \geq \min \left\{N^{\prime}\left(J_{n} F(x)-J_{n} f(x), \frac{t}{2}\right), N^{\prime}\left(J_{n} f(x)-J_{n} F^{\prime}(x), \frac{t}{2}\right)\right\} \\
& \geq \min \left\{N^{\prime}\left(\frac{(F-f)\left(2^{n} x\right)}{2 \cdot 4^{n}}, \frac{t}{8}\right), N^{\prime}\left(\frac{\left(f-F^{\prime}\right)\left(2^{n} x\right)}{2 \cdot 4^{n}}, \frac{t}{8}\right),\right. \\
& \quad N^{\prime}\left(\frac{(F-f)\left(-2^{n} x\right)}{2 \cdot 4^{n}}, \frac{t}{8}\right), N^{\prime}\left(\frac{\left(f-F^{\prime}\right)\left(-2^{n} x\right)}{2 \cdot 4^{n}}, \frac{t}{8}\right), \\
& \quad N^{\prime}\left(\frac{(F-f)\left(2^{n} x\right)}{2 \cdot 2^{n}}, \frac{t}{8}\right), N^{\prime}\left(\frac{\left(f-F^{\prime}\right)\left(2^{n} x\right)}{2 \cdot 2^{n}}, \frac{t}{8}\right), \\
& \left.\quad N^{\prime}\left(\frac{(F-f)\left(-2^{n} x\right)}{2 \cdot 2^{n}}, \frac{t}{8}\right), N^{\prime}\left(\frac{\left(f-F^{\prime}\right)\left(-2^{n} x\right)}{2 \cdot 2^{n}}, \frac{t}{8}\right)\right\} \\
& \geq \sup _{t^{\prime}<t}\left(x, 2^{(q-1) n-3 q}\left(2-2^{p}\right)^{q} t^{\prime q}\right)
\end{aligned}
$$

for all $x \in X$ and all $n \in \mathbb{N}$. Observe that, for $q=\frac{1}{p}>1$, the last term of the above inequality tends to 1 as $n \rightarrow \infty$ by the fuzzy normed property (N5). This implies that $N^{\prime}\left(F(x)-F^{\prime}(x), t\right)=1$ and so we get 
$F(x)=F^{\prime}(x)$ for all $x \in X$ by the fuzzy normed property (N2), which completes the uniqueness. by

Case 2. Let $\frac{1}{2}<q<1$ and let $J_{n} f: X \rightarrow Y$ be a mapping defined

$$
J_{n} f(x)=\frac{1}{2}\left[4^{-n}\left(f\left(2^{n} x\right)+f\left(-2^{n} x\right)\right)+2^{n}\left(f\left(\frac{x}{2^{n}}\right)-f\left(-\frac{x}{2^{n}}\right)\right)\right]
$$

for all $x \in X$. Then we have $J_{0} f(x)=f(x)$ and

$$
\begin{aligned}
J_{j} f(x)-J_{j+1} f(x)= & \frac{-1}{2 \cdot 4^{j+1}} D f\left(2^{j} x, 2^{j} x\right)-\frac{1}{2 \cdot 4^{j+1}} D f\left(-2^{j} x,-2^{j} x\right) \\
& +2^{j-1} D f\left(\frac{x}{2^{j+1}}, \frac{x}{2^{j+1}}\right)-2^{j-1} D f\left(\frac{-x}{2^{j+1}}, \frac{-x}{2^{j+1}}\right)
\end{aligned}
$$

for all $x \in X$ and all $j \geq 0$. If $n+m>m \geq 0$, then we have

$$
\begin{aligned}
& N^{\prime}\left(J_{m} f(x)-J_{n+m} f(x), \sum_{j=m}^{n+m-1}\left(\frac{1}{2}\left(\frac{2^{p}}{4}\right)^{j}+\frac{2}{2^{p}}\left(\frac{2}{2^{p}}\right)^{j}\right) t^{p}\right) \\
& \geq \min \bigcup_{j=m}^{n+m-1}\left\{\operatorname { m i n } \left\{N^{\prime}\left(-\frac{D f\left(2^{j} x, 2^{j} x\right)}{2 \cdot 4^{j+1}}, \frac{2^{j p} t^{p}}{4^{j+1}}\right),\right.\right. \\
& N^{\prime}\left(-\frac{D f\left(-2^{j} x,-2^{j} x\right)}{2 \cdot 4^{j+1}}, \frac{2^{j p} t^{p}}{4^{j+1}}\right), \\
& N^{\prime}\left(2^{j-1} D f\left(\frac{x}{2^{j+1}}, \frac{x}{2^{j+1}}\right), \frac{2^{j} t^{p}}{2^{(j+1) p}}\right), \\
& \left.\left.N^{\prime}\left(-2^{j-1} D f\left(-\frac{x}{2^{j+1}},-\frac{x}{2^{j+1}}\right), \frac{2^{j} t^{p}}{2^{(j+1) p}}\right)\right\}\right\} \\
& \geq \min \bigcup_{j=m}^{n+m-1}\left\{N\left(2^{j} x, 2^{j} t\right), N\left(\frac{x}{2^{j+1}}, \frac{t}{2^{j+1}}\right)\right\}
\end{aligned}
$$

for all $x \in X$ and all $t>0$. By the similar argument to $\left(^{*}\right)$ of the previous case, we can define the limit $F(x):=N^{\prime}-\lim _{n \rightarrow \infty} J_{n} f(x)$ of the Cauchy sequence $\left\{J_{n} f(x)\right\}$ in the fuzzy Banach space $Y$. Moreover, putting $m=0$ in the above inequality, we have

$$
\begin{aligned}
& N^{\prime}\left(f(x)-J_{n} f(x), t\right) \\
& \geq N\left(x, \frac{t^{q}}{\left(\sum_{j=0}^{n-1}\left(\frac{1}{2}\left(\frac{2^{p}}{4}\right)^{j}+\frac{2}{2^{p}}\left(\frac{2}{2^{p}}\right)^{j}\right)\right)^{q}}\right)
\end{aligned}
$$


for each $x \in X$ and $t>0$. To prove that $F$ is a quadratic-additive function, it is enough to show that the last term of (2.5) tends to 1 as $n \rightarrow \infty$ similarly as in Case 1 . By (N3) and (2.1), we get

$$
\begin{aligned}
& N^{\prime}\left(D J_{n} f(x, y), \frac{t}{2}\right) \\
& \geq \min \left\{N^{\prime}\left(\frac{D f\left(2^{n} x, 2^{n} y\right)}{2 \cdot 4^{n}}, \frac{t}{8}\right), N^{\prime}\left(\frac{D f\left(-2^{n} x,-2^{n} y\right.}{2 \cdot 4^{n}}, \frac{t}{8}\right),\right. \\
& \left.\quad N^{\prime}\left(2^{n-1} D f\left(\frac{x}{2^{n}}, \frac{y}{2^{n}}\right), \frac{t}{8}\right), N^{\prime}\left(2^{n-1} D f\left(\frac{-x}{2^{n}}, \frac{-y}{2^{n}}\right), \frac{t}{8}\right)\right\} \\
& \geq \min \left\{N\left(x, 2^{(2 q-1) n-3 q} t^{q}\right), N\left(y, 2^{(2 q-1) n-3 q} t^{q}\right),\right. \\
& \left.\quad N\left(x, 2^{(1-q) n-3 q} t^{q}\right), N\left(y, 2^{(1-q) n-3 q} t^{q}\right)\right\}
\end{aligned}
$$

for each $x, y \in X$ and all $t>0$. Observe that all the terms on the right hand side of the above inequality tend to 1 as $n \rightarrow \infty$ because of $\frac{1}{2}<q<1$. Hence, by the similar argument after (2.5), we can verify that $D F(x, y)=0$ for all $x, y \in X$. Recalling that the inequality (2.2) follows from (2.4) in Case 1, we get the approximation (2.2) from (2.7) in this case by using the same argument.

Now to prove the uniqueness of $F$, let $F^{\prime}$ be another quadraticadditive mapping satisfying the inequality (2.2). Then, together with (N4), (2.2), and the inequality (2.6), we have

$$
\begin{aligned}
& N^{\prime}\left(F(x)-F^{\prime}(x), t\right)=N^{\prime}\left(J_{n} F(x)-J_{n} F^{\prime}(x), t\right) \\
& \geq \min \left\{N^{\prime}\left(J_{n} F(x)-J_{n} f(x), \frac{t}{2}\right), N^{\prime}\left(J_{n} f(x)-J_{n} F^{\prime}(x), \frac{t}{2}\right)\right\} \\
& \geq \min \left\{N^{\prime}\left(\frac{(F-f)\left(2^{n} x\right)}{2 \cdot 4^{n}}, \frac{t}{8}\right), N^{\prime}\left(\frac{\left(f-F^{\prime}\right)\left(2^{n} x\right)}{2 \cdot 4^{n}}, \frac{t}{8}\right),\right. \\
& N^{\prime}\left(\frac{(F-f)\left(-2^{n} x\right)}{2 \cdot 4^{n}}, \frac{t}{8}\right), N^{\prime}\left(\frac{\left(f-F^{\prime}\right)\left(-2^{n} x\right)}{2 \cdot 4^{n}}, \frac{t}{8}\right), \\
& N^{\prime}\left(2^{n-1}\left((F-f)\left(\frac{x}{2^{n}}\right)\right), \frac{t}{8}\right), N^{\prime}\left(2^{n-1}\left(\left(f-F^{\prime}\right)\left(\frac{x}{2^{n}}\right)\right), \frac{t}{8}\right), \\
& \left.N^{\prime}\left(2^{n-1}\left((F-f)\left(\frac{-x}{2^{n}}\right)\right), \frac{t}{8}\right), N^{\prime}\left(2^{n-1}\left(\left(f-F^{\prime}\right)\left(\frac{-x}{2^{n}}\right)\right), \frac{t}{8}\right)\right\} \\
& \geq \min \left\{\sup _{t^{\prime}<t} N\left(x, 2^{(2 q-1) n-2 q}\left(4^{-1}\left(4-2^{p}\right)\left(2^{p}-2\right)\right)^{q} t^{\prime q}\right),\right. \\
& \left.\quad \sup _{t^{\prime}<t} N\left(x, 2^{(1-q) n-2 q}\left(4^{-1}\left(4-2^{p}\right)\left(2^{p}-2\right)\right)^{q} t^{\prime q}\right)\right\}
\end{aligned}
$$


for all $x \in X$ and all $n \in \mathbb{N}$. Since

$$
\lim _{n \rightarrow \infty} 2^{(2 q-1) n-2 q}=\lim _{n \rightarrow \infty} 2^{(1-q) n-2 q}=\infty
$$

in this case, both terms on the right hand side of the above inequality tend to 1 as $n \rightarrow \infty$ by (N5). This implies that $N^{\prime}\left(F(x)-F^{\prime}(x), t\right)=$ 1 and so $F(x)=F^{\prime}(x)$ for all $x \in X$ by (N2), which completes the uniqueness.

Case 3. Finally, we take $0<q<\frac{1}{2}$ and define $J_{n} f: X \rightarrow Y$ by

$J_{n} f(x)=\frac{1}{2}\left[4^{n}\left(f\left(2^{-n} x\right)+f\left(-2^{-n} x\right)\right)+2^{n}\left(f\left(\frac{x}{2^{n}}\right)-f\left(-\frac{x}{2^{n}}\right)\right)\right]$

for all $x \in X$. Then we have $J_{0} f(x)=f(x)$ and

$$
\begin{aligned}
J_{j} f(x)-J_{j+1} f(x)=(2 & \left.\cdot 4^{j-1}+2^{j-1}\right) D f\left(\frac{x}{2^{j+1}}, \frac{x}{2^{j+1}}\right) \\
& +\left(2 \cdot 4^{j-1}-2^{j-1}\right) D f\left(\frac{-x}{2^{j+1}}, \frac{-x}{2^{j+1}}\right),
\end{aligned}
$$

which implies that if $n+m>m \geq 0$, then

$$
\begin{aligned}
& N^{\prime}\left(J_{m} f(x)-J_{n+m} f(x), 2 \sum_{j=m}^{n+m-1}\left(\frac{4}{2^{p}}\right)^{j} \frac{t^{p}}{2^{p}}\right) \\
& \geq \min \bigcup_{j=m}^{n+m-1}\left\{\operatorname { m i n } \left\{N^{\prime}\left(\left(2 \cdot 4^{j-1}+2^{j-1}\right) D f\left(\frac{x}{2^{j+1}}, \frac{x}{2^{j+1}}\right), \frac{\left(4^{j}+2^{j}\right) t^{p}}{2^{(j+1) p}}\right),\right.\right. \\
& \left.\left.\quad N^{\prime}\left(\left(2 \cdot 4^{j-1}-2^{j-1}\right) D f\left(-\frac{x}{2^{j+1}},-\frac{x}{2^{j+1}}\right), \frac{\left(4^{j}-2^{j}\right) t^{p}}{2^{(j+1) p}}\right)\right\}\right\} \\
& \geq \min \bigcup_{j=m}^{n+m-1}\left\{N\left(\frac{x}{2^{j+1}}, \frac{t}{2^{j+1}}\right)\right\} \\
& =N(x, t)
\end{aligned}
$$

for all $x \in X$ and all $t>0$. Similar to the previous cases, it leads us to define a mapping $F: X \rightarrow Y$ given by $F(x):=N^{\prime}-\lim _{n \rightarrow \infty} J_{n} f(x)$. Putting $m=0$ in the last inequality, we have

$$
N^{\prime}\left(f(x)-J_{n} f(x), t\right) \geq N\left(x, \frac{t^{q}}{\left(\frac{2}{2^{p}} \sum_{j=0}^{n-1}\left(\frac{4}{2^{p}}\right)^{j}\right)^{q}}\right)
$$


for all $x \in X$ and all $t>0$. We notice that

$$
\begin{aligned}
& N^{\prime}\left(D J_{n} f(x, y), \frac{t}{2}\right) \\
& \geq \min \left\{N^{\prime}\left(\frac{4^{n}}{2} D f\left(\frac{x}{2^{n}}, \frac{y}{2^{n}}\right), \frac{t}{8}\right), N^{\prime}\left(\frac{4^{n}}{2} D f\left(\frac{-x}{2^{n}}, \frac{-y}{2^{n}}\right), \frac{t}{8}\right)\right. \\
& \left.\quad N^{\prime}\left(2^{n-1} D f\left(\frac{x}{2^{n}}, \frac{y}{2^{n}}\right), \frac{t}{8}\right), N^{\prime}\left(2^{n-1} D f\left(\frac{-x}{2^{n}}, \frac{-y}{2^{n}}\right), \frac{t}{8}\right)\right\} \\
& \geq \min \left\{N\left(x, 2^{(1-2 q) n-3 q} t^{q}\right), N\left(y, 2^{(1-2 q) n-3 q} t^{q}\right),\right. \\
& \left.N\left(x, 2^{(1-q) n-3 q} t^{q}\right), N\left(y, 2^{(1-q) n-3 q} t^{q}\right)\right\}
\end{aligned}
$$

for each $x, y \in X$ and all $t>0$. Since $0<q<\frac{1}{2}$, both terms on the right hand side tend to 1 as $n \rightarrow \infty$, which implies that the last term of (2.5) applied to this case tends to 1 as $n \rightarrow \infty$. Therefore, we can say that $D F \equiv 0$. Moreover, using the similar argument after (2.5) in Case 1, we get the inequality (2.2) from (2.8) in this case. To prove the uniqueness of $F$, let $F^{\prime}: X \rightarrow Y$ be another quadratic-additive function satisfying the inequality (2.2). Then, we get from use of (2.6)

$$
\begin{aligned}
& N^{\prime}\left(F(x)-F^{\prime}(x), t\right) \\
& \geq \min \left\{N^{\prime}\left(J_{n} F(x)-J_{n} f(x), \frac{t}{2}\right), N^{\prime}\left(J_{n} f(x)-J_{n} F^{\prime}(x), \frac{t}{2}\right)\right\} \\
& \geq \min \left\{N^{\prime}\left(\frac{4^{n}}{2}\left((F-f)\left(\frac{x}{2^{n}}\right)\right), \frac{t}{8}\right), N^{\prime}\left(\frac{4^{n}}{2}\left(\left(f-F^{\prime}\right)\left(\frac{x}{2^{n}}\right)\right), \frac{t}{8}\right),\right. \\
& \quad N^{\prime}\left(\frac{4^{n}}{2}\left((F-f)\left(\frac{-x}{2^{n}}\right)\right), \frac{t}{8}\right), N^{\prime}\left(\frac{4^{n}}{2}\left(\left(f-F^{\prime}\right)\left(\frac{-x}{2^{n}}\right)\right), \frac{t}{8}\right), \\
& \quad N^{\prime}\left(2^{n-1}\left((F-f)\left(\frac{x}{2^{n}}\right)\right), \frac{t}{8}\right), N^{\prime}\left(2^{n-1}\left(\left(f-F^{\prime}\right)\left(\frac{x}{2^{n}}\right)\right), \frac{t}{8}\right), \\
& \left.\quad N^{\prime}\left(2^{n-1}\left((F-f)\left(\frac{-x}{2^{n}}\right)\right), \frac{t}{8}\right), N^{\prime}\left(2^{n-1}\left(\left(f-F^{\prime}\right)\left(\frac{-x}{2^{n}}\right)\right), \frac{t}{8}\right)\right\} \\
& \geq \sup _{t^{\prime}<t} N\left(x, 2^{(1-2 q) n-3 q}\left(2^{p}-4\right)^{q} t^{\prime q}\right)
\end{aligned}
$$

for all $x \in X$ and all $n \in \mathbb{N}$. Observe that, for $0<q<\frac{1}{2}$, the last term tends to 1 as $n \rightarrow \infty$ by the fuzzy normed property (N5). This implies that $N^{\prime}\left(F(x)-F^{\prime}(x), t\right)=1$ and $F(x)=F^{\prime}(x)$ for all $x \in X$ by the fuzzy normed property (N2), completing the uniqueness. 
Remark 2.2. Consider a mapping $f: X \rightarrow Y$ satisfying (2.1) for all $x, y \in X$ and a real number $q<0$. Take any $t>0$. If we choose a real number $s$ with $0<2 s<t$, then we have

$$
N^{\prime}(D f(x, y), t) \geq N^{\prime}(D f(x, y), 2 s) \geq \min \left\{N\left(x, s^{q}\right), N\left(y, s^{q}\right)\right\}
$$

for all $x, y \in X$. Since $q<0$, we have $\lim _{s \rightarrow 0^{+}} s^{q}=\infty$. This implies that

$$
\lim _{s \rightarrow 0^{+}} N\left(x, s^{q}\right)=\lim _{s \rightarrow 0^{+}} N\left(y, s^{q}\right)=1
$$

and so

$$
N^{\prime}(D f(x, y), t)=1
$$

for all $x, y \in X$ and all $t>0$. By (N2), it allows us to get $D f(x, y)=0$ for all $x, y \in X$. In other words, $f$ is itself a quadratic additive mapping if $f$ is a fuzzy $q$-almost quadratic-additive mapping for the case $q<0$.

Corollary 2.3. Let $f$ be an even mapping satisfying all of the conditions of Theorem 2.1. Then there is a unique quadratic mapping $F: X \rightarrow Y$ such that

$$
N^{\prime}(F(x)-f(x), t) \geq \sup _{t^{\prime}<t} N\left(x,\left(2^{-1}\left|4-2^{p}\right| t^{\prime}\right)^{q}\right)
$$

for all $x \in X$ and $t>0$, where $p:=1 / q$.

Proof. Let $J_{n} f$ be defined as in Theorem 2.1. Since $f$ is an even mapping, we obtain

$$
J_{n} f(x)= \begin{cases}\frac{f\left(2^{n} x\right)+f\left(-2^{n} x\right)}{2 \cdot 4^{n}}, & \text { if } 0<q<\frac{1}{2} \\ \frac{1}{2}\left[4^{n}\left(f\left(2^{-n} x\right)+f\left(-2^{-n} x\right)\right)\right], & \text { if } q>\frac{1}{2}\end{cases}
$$

for all $x \in X$. Notice that $J_{0} f(x)=f(x)$ and

$$
J_{j} f(x)-J_{j+1} f(x)= \begin{cases}\frac{-D f\left(2^{j} x, 2^{j} x\right)}{4^{j+1}}, & \text { if } 0<q<\frac{x}{2} ; \\ 4^{j} D f\left(\frac{x}{2^{j+1}}, \frac{x}{2^{j+1}}\right), & \text { if } q>\frac{1}{2}\end{cases}
$$

for all $x \in X$ and $j \in \mathbb{N} \cup\{0\}$. From these, using the similar method in Theorem 2.1, we obtain the quadratic-additive mapping $F$ satisfying the fuzzy inequality (2.9). Notice that $F$ is also even, $F(x):=N^{\prime}-$ $\lim _{n \rightarrow \infty} J_{n} f(x)$ for all $x \in X$, and $D F(x, y)=0$ for all $x, y \in X$. Hence, we get

$$
F(x+y)+F(x-y)-2 F(x)-2 F(y)=D F(x, y)-D F(0, y)=0
$$

for all $x, y \in X$. This means that $F$ is in fact a quadratic mapping. 
Corollary 2.4. Let $f$ be an odd mapping satisfying all of the conditions of Theorem 2.1. Then there is a unique additive mapping $F: X \rightarrow Y$ such that

$$
N^{\prime}(F(x)-f(x), t) \geq \sup _{t^{\prime}<t} N\left(x,\left(2^{-1}\left|2-2^{p}\right| t^{\prime}\right)^{q}\right)
$$

for all $x \in X$ and $t>0$, where $p=1 / q$.

Proof. Let $J_{n} f$ be defined as in Theorem 2.1. Since $f$ is an odd mapping, we obtain

$$
J_{n} f(x)= \begin{cases}\frac{f\left(2^{n} x\right)-f\left(-2^{n} x\right)}{2^{n+1}}, & \text { if } 0<q<1 \\ 2^{n-1}\left[f\left(2^{-n} x\right)-f\left(-2^{-n} x\right)\right], & \text { if } q>1\end{cases}
$$

for all $x \in X$. Notice that $J_{0} f(x)=f(x)$ and

$$
J_{j} f(x)-J_{j+1} f(x)=\left\{\begin{array}{c}
\frac{1}{2^{j+2}}\left[D f\left(-2^{j} x,-2^{j} x\right)-D f\left(2^{j} x, 2^{j} x\right)\right], \\
\text { if } 0<q<1 ; \\
2^{j-1}\left[D f\left(\frac{x}{2^{j+1}}, \frac{x}{2^{j+1}}\right)-D f\left(\frac{-x}{2^{j+1}}, \frac{-x}{2^{j+1}}\right)\right], \\
\text { if } q>1
\end{array}\right.
$$

for all $x \in X$ and $j \in \mathbb{N} \cup\{0\}$. From these, using the similar method in Theorem 2.1, we obtain the quadratic-additive mapping $F$ satisfying (2.10). Notice that $F$ is also odd, $F(x):=N^{\prime}-\lim _{n \rightarrow \infty} J_{n} f(x)$ for all $x \in X$, and $D F(x, y)=0$ for all $x, y \in X$. Hence, we get

$$
F(x+y)-F(x)-F(y)=\frac{1}{2} D F(x, y)=0
$$

for all $x, y \in X$. This means that $F$ is in fact an additive mapping.

We can use Theorem 2.1 to get a classical result in the framework of normed spaces as follows. Let $(X,\|\cdot\|)$ be a normed linear space. Then we can define a fuzzy norm $N_{X}$ on $X$ by following

$$
N_{X}(x, t)= \begin{cases}0, & t \leq\|x\| \\ 1, & t>\|x\|\end{cases}
$$

where $x \in X$ and all $t \in \mathbb{R}$, see [11] for details. Suppose that $f: X \rightarrow Y$ is a mapping into a Banach space $(Y,\||\cdot|\|)$ such that

$$
\text { ||Df( } x, y)\left|\|\mid \leq\| x\left\|^{p}+\right\| y \|^{p}\right.
$$

for all $x, y \in X$, where $p>0$ and $p \neq 1,2$. Let $N_{Y}$ be a fuzzy norm on $Y$ defined by above as usual. Then we get

$$
N_{Y}(D f(x, y), s+t)= \begin{cases}0, & s+t \leq\|\mid\| D f(x, y) \| \\ 1, & s+t>\|\mid\| D f(x, y) \|\end{cases}
$$


for all $x, y \in X$ and all $s, t \in \mathbb{R}$. Consider the case $N_{Y}(D f(x, y), s+t)=$ 0 . This implies that

$$
\|x\|^{p}+\|y\|^{p} \geq\||D f(x, y) \|| \geq s+t
$$

and so either $\|x\|^{p} \geq s$ or $\|y\|^{p} \geq t$ in this case. Hence, for $q=\frac{1}{p}$, we have

$$
\min \left\{N_{X}\left(x, s^{q}\right), N_{X}\left(y, t^{q}\right)\right\}=0
$$

for all $x, y \in X$ and $s, t>0$. Therefore, in any case, the inequality

$$
N_{Y}(D f(x, y), s+t) \geq \min \left\{N_{X}\left(x, s^{q}\right), N_{X}\left(y, t^{q}\right)\right\}
$$

holds. It means that $f$ is a fuzzy $q$-almost quadratic-additive mapping, and by Theorem 2.1, we get the following stability result.

Corollary 2.5. Let $(X,\|\cdot\|)$ be a normed linear space and let $(Y,|||\cdot| \mid)$ be a Banach space. If a mapping $f$ satisfies the functional inequality

$$
\text { ||Df(x,y)||| } \leq\|x\|^{p}+\|y\|^{p}
$$

for all $x, y \in X$, where $p>0$ and $p \neq 1,2$, then there is a unique quadratic-additive mapping $F: X \rightarrow Y$ such that

$$
|\|F(x)-f(x)\|| \leq \begin{cases}\frac{2\|x\|^{p}}{2-2^{p}}, & \text { if } 0<p<1 ; \\ \frac{4\|x\|^{p}}{\left(2^{p}-2\right)\left(4-2^{p}\right)}, & \text { if } 1<p<2 ; \\ \frac{2\|x\|^{p}}{2^{p}-4}, & \text { if } 2<p\end{cases}
$$

for all $x \in X$.

\section{References}

[1] T. Aoki, On the stability of the linear transformation in Banach spaces, J. Math. Soc. Japan 2 (1950), 64-66.

[2] T. Bag and S. K. Samanta, Finite dimensional fuzzy normed linear spaces, J. fuzzy Math. 11 (2003), no. 3, 687-705.

[3] S. C. Cheng and J. N. Mordeson, Fuzzy linear operator and fuzzy normed linear spaces, Bull. Calcutta Math. Soc. 86 (1994), 429-436.

[4] S. Czerwik, On the stability of the quadratic mapping in normed spaces, Abh. Math. Sem. Univ. Hamburg 62 (1992), 59-64.

[5] D. H. Hyers, On the stability of the linear functional equation, Proc. Natl. Acad. Sci. USA 27 (1941), 222-224.

[6] K. W. Jun and Y. H. Lee, A generalization of the Hyers-Ulam-Rassias stability of the Pexiderized quadratic equations II, Kyungpook Math. J. 47 (2007), 91103.

[7] S. M. Jung and P. K. Sahoo, Stability of a functional equation of Drygas, Aequationes Math. 64 (2002), 263-273. 
[8] A. K. Katsaras, Fuzzy topological vector spaces II, Fuzzy Sets and Systems 12 (1984), 143-154.

[9] I. Kramosil and J. Michalek, Fuzzy metric and statistical metric spaces, Kybernetica 11 (1975), 326-334.

[10] Y. H. Lee, On the Hyers-Ulam-Rassias stability of the generalized polynomial function of degree 2, J. Chuncheong Math. Soc. 22 (2009), 201-209.

[11] A. K. Mirmostafaee and M. S. Moslehian, Fuzzy almost quadratic functions, Results Math. 52 (2008), 161-177.

[12] A. K. Mirmostafaee and M. S. Moslehian, Fuzzy versions of Hyers-UlamRassias theorem, Fuzzy Sets and Systems 159 (2008), 720-729.

[13] C. G. Park, On the stability of the Cauchy additive and quadratic type functional equation, Bull. Korean Math. Soc. preprint.

[14] Th. M. Rassias, On the stability of the linear mapping in Banach spaces, Proc. Amer. Math. Soc. 72 ( 1978), 297-300.

[15] F. Skof, Local properties and approximations of operators, Rend. Sem. Mat. Fis. Milano 53 (1983), 113-129.

[16] S. M. Ulam, A collection of mathematical problems, Interscience, New York, 1968.

[17] D. L. Yang, Remarks on the stability of Drygas equation and the Pexiderquadratic equation, Aequationes Math. 68 (2004), 108-116.

Department of Mathematics

Chungnam National University

Daejeon 305-764, Republic of Korea

E-mail: hmkim@cnu.ac.kr

$* *$

Department of Mathematics Education

Gongju National University of Education

Gongju 314-711, Republic of Korea

E-mail: yanghi2@hanmail.net 